\title{
Psicologia e Educação Inclusiva no Brasil na Perspectiva do Periódico Psicologia: Ciência e Profissão
}

Psychology and Inclusive Education in Brazil in the Journal Psicologia: Ciência e Profissão

Psicología y Educación Inclusiva en Brasil Bajo la Perspectiva del Periódico Psicologia: Ciência e Profissão

Érika Lourenço, Ciléia Saori Hamada de Miranda \& Jordana Mendes Póvoa

Universidade Federal de Minas Gerais

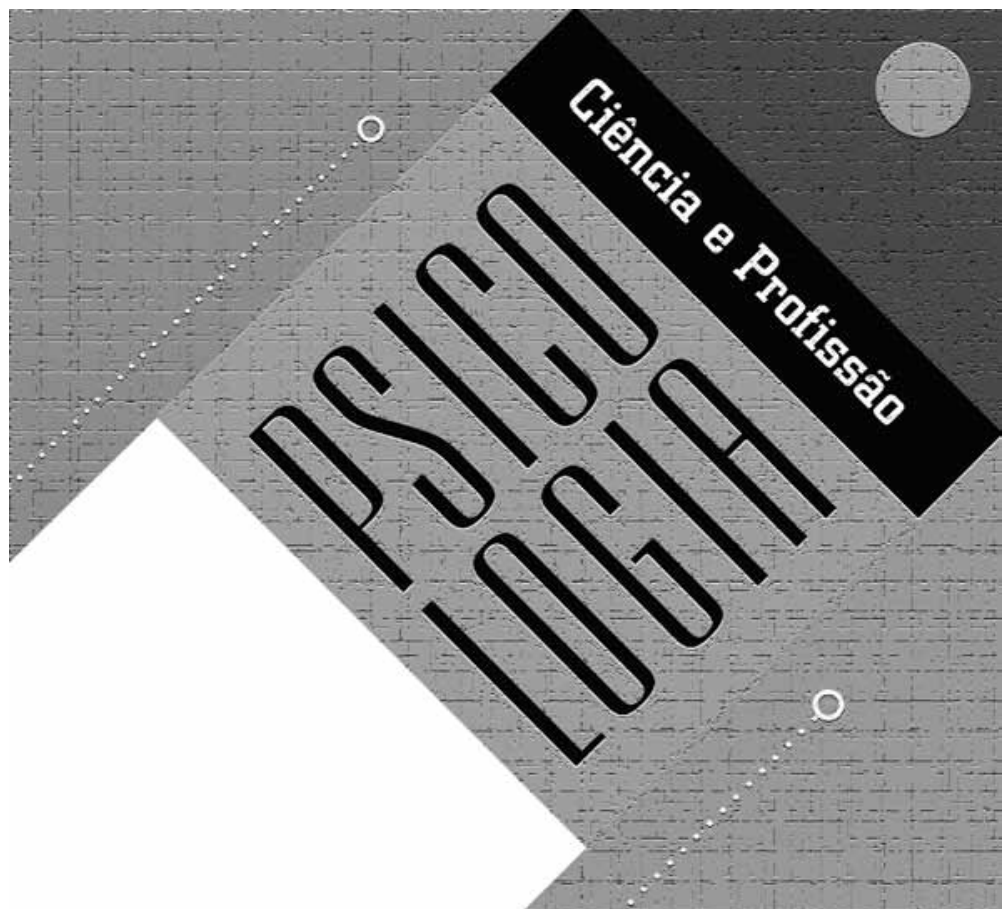


Resumo: A presente pesquisa tem como objetivo investigar as relações entre a Psicologia e a educação inclusiva no Brasil a partir do periódico Psicologia: Ciência e Profissão. Desde sua criação, em 1979, esse periódico foi apresentado como uma publicação científica e profissional que visava a representar o pensamento nacional em Psicologia. Com isso, pode-se considerar que também expressa de maneira representativa as concepções teóricas e as práticas dos profissionais da área ao longo do tempo. Para a seleção dos artigos referentes à área de interesse da pesquisa, foram considerados título, resumo e palavras-chave dos artigos publicados em todos os números do periódico. Os artigos selecionados foram analisados segundo as seguintes categorias: autoria, tipo de artigo, referencial adotado e tendências temáticas. Os resultados revelaram que os artigos se dividem quase igualmente entre teóricos e práticos, que seus autores são em maioria afiliados a instituições públicas e que a Psicologia social é o principal referencial adotado. Concluise que, embora seja pequeno, o número de artigos sobre as relações entre Psicologia e educação inclusiva ao longo da história do periódico, esse número vem se tornando mais expressivo nos últimos anos, e que, para uma visão mais realista do tema, é necessário recorrer a fontes complementares.

Palavras-chave: Educação inclusiva. Psicologia. Periódicos científicos. História da Psicologia-Brasil.

Abstract: The current research aims to study the relations between psychology and inclusive education in Brazil in accordance with the journal Psicologia: Ciência e Profissão. Since its foundation in 1979, this journal was introduced as a scientific and professional publication that aimed at framing the national thought on psychology. With that in mind, one can assume that the journal also expresses, in a meaningful way, the theoretical concepts and the expert's practices throughout the time. Titles, summaries and keywords published in all the volumes of the journal were taken into consideration when selecting the articles which matched the area of interest of the research. The selected articles were analyzed according to the following categories: authorship, type of article, adopted references and thematic tendencies. The results showed that the articles are split almost equally between theoretical and practical, most of the authors are linked to public institutions and social psychology is the main chosen reference. One comes to the conclusion that along the history of the journal, although the number of articles on the relation between psychology and inclusive education throughout the history of the journal proves to be small, this number has become more expressive in the last years, and that in order to have a more realistic view of the subject it is required to search for other complementary sources.

Keywords: Inclusive education. Psychology. Scientific Journal. History of psychology- Brazil.

Resumen: La presente pesquisa tiene como objetivo investigar las relaciones entre la Psicología y la educación inclusiva en el Brasil a partir del periódico Psicología: Ciencia y Profesión. Desde su creación, en 1979, ese periódico fue presentado como una publicación científica y profesional que visaba a representar el pensamiento nacional en Psicología. Con eso, se puede considerar que también expresa de manera representativa las concepciones teóricas y las prácticas de los profesionales del área a lo largo del tiempo. Para la selección de los artículos referentes al área de interés de la pesquisa, fueron considerados título, resumen y palabras clave de los artículos publicados en todos los números del periódico. Los artículos seleccionados fueron analizados según las siguientes categorías: autoría, tipo de artículo, referencial adoptado y tendencias temáticas. Los resultados revelaron que los artículos se dividen casi igualmente entre teóricos y prácticos, que sus autores son en mayoría afiliados a instituciones públicas y que la Psicología social es el principal referencial adoptado. Se concluye que, aunque sea pequeño, el número de artículos sobre las relaciones entre Psicología y educación inclusiva a lo largo de la historia del periódico, ese número viene tornándose más expresivo en los últimos años, y que, para una visión más realista del tema, es necesario recurrir a fuentes complementarias.

Palabras clave: Educación inclusiva. Psicología. Periódico cientifíco. Historia de la Psicología-Brasil.

\section{A educação inclusiva diz respeito à capacidade} das escolas para acolher e educar crianças, jovens e adultos sem qualquer tipo de exclusão. No Brasil, os movimentos pela educação inclusiva ganharam força a partir da década de 1980, com a divulgação de dados alarmantes sobre o fracasso, a evasão e a repetência escolar e com o aumento de demandas para a criação de classes e escolas especiais.
Reivindicações de diversas organizações da sociedade civil e de associações de pessoas com necessidade especiais contribuíram para que, nessa mesma década, a Constituição Federal (promulgada em 1988) incorporasse os ideais da educação para todos e para que as políticas educacionais no país começassem a passar por modificações substanciais (Lima, 2006; Lourenço, 2010). 
Embora, segundo Campos e Bernardes (2005) e Guedes (2010), o periódico tenha passado por uma série de fases e de mudanças definidas por alterações em sua política editorial, pode-se considerar que, pela maturidade que alcançou ao longo dos anos, consolidandose "como um espaço privilegiado de divulgação dos trabalhos produzidos por psicólogos no País".
A partir da década de 1990, as discussões e as propostas pela educação inclusiva no Brasil se ampliaram e começaram a concretizarse através da Lei de Diretrizes e Bases da Educação Nacional, aprovada em 1996, do Plano Nacional da Educação, aprovado em 2001, dos Planos Nacionais da Educação para os Direitos Humanos, aprovados respectivamente em 2003 e em 2006, e de programas e planos governamentais como o Programa Educação Inclusiva: direito à diversidade, implementado em 2003, Projeto Educar na Diversidade, desenvolvido entre 2005 e 2006, e o Plano de Metas Compromisso Todos pela Educação, aprovado em 2007.

Todas essas iniciativas causaram um impacto significativo na tradicional organização dos sistemas escolares, e ainda hoje representam um desafio tanto para os educadores como para os demais profissionais que atuam no contexto escolar, como os psicólogos.

O objetivo deste estudo foi investigar as relações entre Psicologia e educação inclusiva a partir do periódico Psicologia: Ciência e Profissão. Esse periódico foi escolhido porque, desde a sua fundação, em 1979, se propôs ser uma "publicação de natureza científica e profissional (...) representando o pensamento nacional em Psicologia, pela expressão de suas várias linhas de pesquisa, abordagens e tendências, tanto em seus aspectos teóricos como nos metodológicos" (Saldanha, 1979, s/p.). Embora, segundo Campos e Bernardes (2005) e Guedes (2010), o periódico tenha passado por uma série de fases e de mudanças definidas por alterações em sua política editorial, pode-se considerar que, pela maturidade que alcançou ao longo dos anos, consolidando-se "como um espaço privilegiado de divulgação dos trabalhos produzidos por psicólogos no País" (Campos, 2005, p. 510), representa, em grande medida, as tendências de atuação prática e de pesquisa dos psicólogos brasileiros, de maneira geral e, especialmente, no que concerne a sua atuação e às suas investigações em relação à educação inclusiva.

\section{Método}

Foram analisados os artigos publicados no periódico Psicologia: Ciência e Profissão que apresentavam um olhar da Psicologia sobre questões relacionadas à educação inclusiva. Para a seleção de artigos para análise, foram consultados todos os números do periódico, desde aquele identificado como número 0, publicado em 1979, até o número 1 do volume 32, publicado em 2012. Foram incluídos na pesquisa inicialmente os artigos que apresentavam os seguintes termos no título, no resumo ou nas palavras-chave: educação inclusiva, inclusão e exclusão. Considerando as relações históricas entre as discussões sobre educação inclusiva e educação especial, foram também incluídos os artigos que apresentavam os termos: classes ou turmas especiais, educação especial, deficiência e altas habilidades/superdotação. Devido ao fato de que apenas os artigos publicados a partir do volume 20 , número 3 do ano 2000, apresentavam resumo e palavras-chave, os artigos publicados antes dessa data foram selecionados levando em conta apenas o título. Quando o conteúdo do artigo selecionado não indicava uma discussão referente à educação inclusiva, o mesmo era desconsiderado. Não foram levados em conta os artigos referentes a homenagens e biografias.

Ao todo, foram selecionados para análise 16 artigos com data de publicação entre os anos 1992 e 2010. Dentre esses artigos, 9 foram publicados entre os anos 1992 e 2000, e 7 foram publicados entre os anos 2007 e 2010. Cada artigo selecionado foi analisado em relação aos seguintes itens: autoria, características do relato, uso de referências e temática. 
No tocante à autoria, foi contado o número de autores por artigo e foi identificado o tipo de parceria em artigos com mais de um autor, se local, nacional ou internacional. Além disso, foi feita a categorização da procedência institucional dos autores em instituição de ensino superior federal, estadual, particular ou outras instituições. Foi também considerada a procedência geográfica dos autores.

Com relação às características, os relatos foram classificados como teóricos, empíricos (com coleta e análise de dados) ou práticos (com relato de experiência profissional). Os artigos empíricos, por sua vez, foram classificados como quantitativos ou qualitativos.

As referências citadas nos artigos foram classificadas por tipo e idioma. Cada referência foi categorizada como livro, capítulo de livro, artigo de periódico científico, dissertação ou tese, resumo publicado ou outros tipos de referência. Foram contadas as referências em língua portuguesa e em língua estrangeira.

As temáticas dos artigos foram analisadas considerando-se os objetivos descritos pelos autores, e foram observados também os referenciais teóricos adotados.

\section{Resultados}

\section{Autoria dos artigos}

Com relação ao número de autores por artigo, 10 dos 16 artigos selecionados tinham apenas um autor, 3 artigos foram escritos por 2 autores, 2 artigos foram publicados por 3 autores, e 1 artigo foi publicado por 6 autores. Até o ano 2000, todos os artigos selecionados tinham autoria individual. A partir do ano 2007, começaram a aparecer artigos escritos por 2 ou mais autores. Todos os artigos com mais de um autor mostram que as parcerias estabelecidas eram locais, ou seja, entre autores da mesma instituição de ensino superior.
Quanto ao tipo de instituição, 5 artigos foram encaminhados por instituições de ensino superior federal, 6 foram escritos por autores de instituição de ensino superior estadual, 3 foram encaminhados por instituições de ensino superior particular, e 2 não identificaram a instituição de origem dos autores. Todos os artigos foram encaminhados por pesquisadores afiliados a universidades, sendo predominante a presença de artigos de pesquisadores de universidades públicas. As universidades de origem dos artigos foram as seguintes: Universidade Federal de Pernambuco - 1 artigo, Universidade Federal de Minas Gerais - 1 artigo, Universidade Federal de Alagoas - 1 artigo, Universidade Federal da Bahia - 1 artigo, Universidade de Brasília - 1 artigo, Universidade de São Paulo - 5 artigos, Universidade do Estado do Rio de Janeiro - 1 artigo, Pontifícia Universidade Católica de São Paulo - 1 artigo, Pontifícia Universidade Católica de Campinas - 1 artigo, Universidade Salgado de Oliveira - 1 artigo. Dentre os 2 artigos que não mencionam a instituição de origem dos autores, 1 deles indica que o autor é professor de Psicologia no ensino superior, enquanto o outro não faz qualquer menção à afiliação institucional de seu autor.

No que concerne à localização geográfica, 10 artigos provêm de universidades localizadas na Região Sudeste (7 de São Paulo, 2 do Rio de Janeiro e 1 de Minas Gerais), 3 artigos provêm da Região Nordeste (1 do Pernambuco, 1 de Alagoas e 1 da Bahia), e 1 artigo provêm do Distrito Federal. Como mencionado no parágrafo anterior, em 2 artigos, não constam a identificação institucional ou geográfica.

\section{Características dos artigos}

Nos 16 artigos identificados, 7 são textos teóricos, 5 são trabalhos empíricos e 4 são práticos. Dentre os artigos empíricos, 3 apresentam um tratamento qualitativo de dados, e 2 apresentam uma análise quantitativa dos dados. 
Os artigos práticos foram publicados entre os anos 1992 e 2000. Já os artigos empíricos foram todos publicados a partir do ano 2007. Os artigos teóricos, por sua vez, estão bem distribuídos entre os anos 1992 e 2000 e entre os anos 2007 e 2010.

Dentre os artigos empíricos quantitativos, um trabalhou com uma amostra de 52 sujeitos, e o outro, com uma amostra de 14 sujeitos.

\section{Uso de referências}

Os 16 artigos somaram 302 referências bibliográficas, indicando a média de 18,9 referências por artigo. No entanto, 203 das referências estão concentradas nos 7 artigos publicados no período entre 2007 e 2010, o que aponta a média de 29 referências por artigo nesse período e a média de 11 referências por artigo no período entre 1992 e 2000.

Ao todo, foram citados 44 artigos científicos impressos, 157 livros, 45 capítulos de livros, 8 teses de doutorado e 12 dissertações de mestrado. As demais referências são de artigos de jornal, legislação, anais de eventos científicos e revistas de variedades e de notícias, de circulação semanal. As referências a artigos científicos aumentaram de 16, no período entre 1992 e 2000, para 28, no período entre 2007 e 2010. As referências a livros, por sua vez, aumentaram de 51, no período entre 1992 e 2000, para 106, entre 2000 e 2010. Já as referências a capítulos de livros passaram de 9, no período até 2000, para 36, no período seguinte. As dissertações de mestrado foram igualmente citadas nos dois períodos, ao passo que as teses de doutorado foram citadas predominantemente no período entre 2007 e 2010.

Das 302 referências citadas, 28 estão em língua estrangeira, sendo 17 em inglês e 11 em espanhol. A maior concentração de referências em língua estrangeira está nos artigos publicados no período entre 2007 e 2010 - 19 referências.

\section{Temáticas dos artigos}

Foram identificadas, nos artigos, 4 grandes categorias temáticas em torno das quais se desenvolvia a discussão sobre educação inclusiva: deficiências, superdotação/altas habilidades, dificuldades de aprendizagem e educação indígena. Em termos numéricos, os artigos estavam distribuídos da seguinte forma entre essas temáticas: deficiências e superdotação/altas habilidades - 14 artigos, dificuldades de aprendizagem - 1 artigo, educação indígena - 1 artigo.

Os artigos que versam sobre a inclusão escolar das pessoas com deficiências (intelectual e/ ou física) estão bem distribuídos ao longo dos períodos em que se concentram. O tema superdotação/altas habilidades é tratado em um artigo em 1992 e novamente em 2010. A educação indígena, no contexto da educação inclusiva, é temática contemplada em 2000, e as dificuldades de aprendizagem e sua relação com a educação inclusiva são abordadas em 2010.

Quanto aos referenciais adotados, nota-se a tendência dos autores a recorrer a referenciais específicos dos temas que estão privilegiando em seus artigos, ou seja, educação inclusiva, educação especial, deficiência física, deficiência mental, superdotação, etc. No campo específico da Psicologia, estão presentes nas referências autores representantes da Psicologia educacional e escolar crítica e da Psicologia social crítica, no entanto, nenhum autor da área recebe destaque em frequência de citações.

\section{Discussão}

Os autores, as características dos artigos e as referências utilizadas

Os resultados encontrados mostram o destaque das instituições públicas (universidades 
federais e estaduais) localizadas na Região Sudeste na produção científica sobre a educação inclusiva. Esses resultados são corroborados por Yamamoto, Souza e Yamamoto (1999) e Costa, Amorim e Costa (2010), que ressaltam os fatos de que a produção científica no Brasil está atrelada ao ambiente acadêmico e, em seu contexto, aos programas de pós-graduação e que a maior parcela de instituições de ensino superior e de programas de pós-graduação no País está concentrada nos Estados da Região Sudeste. Embora, segundo Costa et al., seja evidente a tendência das políticas governamentais no sentido de enfrentar essa disparidade com a interiorização do ensino superior e da pesquisa, esse aspecto ainda não se manifestou nas publicações sobre educação inclusiva no periódico Psicologia: Ciência e Profissão. Também ainda não se mostrou presente nessas publicações a relevância das parcerias interinstitucionais na produção de pesquisas.

A distribuição dos artigos teóricos, práticos e empíricos sobre educação inclusiva ao longo dos anos acompanha tanto uma tendência da Psicologia no Brasil, de modo geral, como as mudanças na política do periódico. Souza, Gauer e Hutz (2004) chamam a atenção para uma tendência da Psicologia brasileira, nas últimas décadas, de se direcionar para a pesquisa e, especialmente, para as pesquisas empíricas, o que pode explicar a predominância dos artigos empíricos sobre educação inclusiva a partir do ano 2007, em detrimento de artigos práticos, com discussões baseadas em experiências profissionais que eram frequentes até o ano 2000 .

Aliada a essa tendência, há que se considerar o impacto das mudanças na política do periódico ao longo do tempo. Campos e Bernardes (2005) relatam as modificações na linha editorial do periódico, identificando quatro fases em sua história. Entre 1979 e 1983, propunha-se a representar o pensamento nacional em Psicologia e dava prioridade a trabalhos sobre história da Psicologia e que divulgassem as pesquisas na área realizadas no Brasil. Entre 1984 e 1991, a política editorial apontava a relevância social dos trabalhos publicados, dando prioridade às reflexões sobre o conhecimento e a prática. Nessa fase, a revista se propôs ser menos acadêmica e mais flexível. De 1992 a 1996, a ênfase editorial estava na história da ciência psicológica, na divulgação de trabalhos considerados socialmente relevantes e, ao mesmo tempo, no atendimento às demandas espontâneas de publicação de artigos científicos e de relatos de experiência profissional. A partir de 1997 até 2004, a revista passa a enfatizar a divulgação de trabalhos de pesquisa de primeira linha, visando a tornar-se um periódico de referência na área. Gomes e Gauer (2010) completam o trabalho de Campos e Bernardes, identificando a fase entre 2004 e 2009 como aquela em que o periódico define sua identidade como revista científica de referência e de grande alcance, inclusive internacional. Assim, verificase que, à medida que se configura como periódico científico, os artigos com relatos de experiências em educação inclusiva vão sendo acompanhados por discussões teóricas mais elaboradas e acabam por ser substituídos por artigos empíricos e/ou teóricos.

Essas mudanças na linha editorial do periódico se refletem também no uso de referências pelos autores dos artigos. Comparando-se os artigos publicados no período entre 1992 e 2000 com aqueles publicados entre 2007 e 2010, nota-se o significativo aumento do número médio de referências por artigo, assim como o crescimento da diversidade das referências adotadas por um mesmo autor. Ao se comparar as referências apresentadas nos dois períodos, fica também evidente o aumento de referências internacionais, o que pode ser identificado como resultado das características mais empíricas e teóricas 
dos artigos e de maior facilidade de acesso a publicações estrangeiras.

\section{Os temas dos artigos}

Observou-se que a maioria dos artigos selecionados (14 dos 16 artigos) versava sobre a inclusão escolar de alunos com necessidades educacionais especiais, ou seja, crianças, jovens e adultos com deficiências, superdotados e talentosos. Embora a divisão dos artigos em categorias temáticas já tenha sido apresentada no item resultados, vale a pena considerá-los de uma maneira um pouco mais específica.

Dos artigos que se referem à inclusão de alunos com necessidades educacionais especiais, 2 abordaram a questão dos estudantes com altas habilidades ou superdotados, discutindo os critérios que vêm sendo adotados para sua identificação, a necessidade de que professores e psicólogos sejam preparados para identificá-los e encaminhá-los para um atendimento especializado e os mitos que dificultam a identificação dos superdotados no contexto escolar.

A temática da inclusão de alunos com necessidades especiais na perspectiva da escola foi contemplada em 5 artigos. Estes consideraram os dilemas e as dificuldades dos professores diante da inclusão desses alunos, as atitudes e os comportamentos dos professores frente à educação inclusiva, a formação de professores para a educação inclusiva e o papel da direção da escola na sua transformação para uma escola inclusiva.

Outro grupo de 5 artigos tratou da educação especial e da educação inclusiva do ponto de vista teórico, definindo a deficiência mental, a normalidade e a patologia, a inclusão e a exclusão, e questiona não apenas esses conceitos mas também as práticas que historicamente têm levado à identificação e à rotulação do aluno como deficiente.
Um dos artigos tratou da inclusão dos alunos com deficiência física, criticando a associação que se costuma fazer entre deficiência física e deficiência intelectual a partir de uma experiência em uma escola que incluía alunos com deficiência física.

Outro artigo discutiu a educação inclusiva no ensino superior, apontando os desafios que as instituições de ensino superior começam a enfrentar à medida que os alunos com necessidades educacionais especiais concluem o ensino médio. O artigo abordou questões como a elegibilidade de alunos com necessidades educacionais especiais para o ensino superior, a formação de professores e como trabalhar com a diferença no cotidiano escolar.

Apenas 2 dos artigos selecionados segundo os critérios propostos para esta pesquisa fugiram da temática da inclusão dos educandos com necessidades educacionais. O primeiro discutiu as possíveis contribuições da Psicologia na educação indígena, e o outro fez referência ao papel do psicólogo escolar diante das dificuldades de aprendizagem, apresentando uma avaliação crítica da Psicologia frente às queixas escolares e ao fracasso escolar.

Os 16 artigos acima descritos têm em comum a preocupação com a definição dos termos adotados (inclusão, educação inclusiva, deficiência, etc) e uma postura crítica diante da educação inclusiva. A postura crítica se revela não apenas em uma crítica fundamentada nos conceitos de inclusão e nas práticas inclusivas adotadas pelas escolas, mas também se mostra na crítica ao papel do psicólogo escolar diante dos dilemas, impasses e dificuldades gerados pela educação inclusiva e na análise do papel dos educadores na transformação das escolas regulares em escolas inclusivas.

A presença desses artigos no período entre 1992 e 2010, com sua frequência 
concentrada no intervalo entre 1992-2000 e depois no intervalo entre 2007-2010, pode ser relacionada à própria trajetória dos movimentos sociais e às políticas públicas pela educação inclusiva.

Ao longo da década de 90, o Brasil viu a ampliação das discussões e das propostas pela educação inclusiva, evidenciando as iniciativas da sociedade civil e do Estado no sentido de buscar cumprir os compromissos assumidos nos encontros promovidos pela UNESCO, em 1990, para tratar da Educação para Todos. Em 1996, a Lei de Diretrizes e Bases da Educação Nacional reafirmou a educação como direito de todos, instituiu a Década da Educação, com início em 1997, e definiu a educação especial como modalidade escolar a ser oferecida preferencialmente na rede regular. Em 2001, foi aprovado o Plano Nacional de Educação, e, nos anos 2003 e 2006, foram aprovados os Planos Nacionais de Educação em Direitos Humanos. Esses documentos e outros que a eles se seguiram propuseram, dentre outras coisas, que se orientasse as políticas educacionais para uma cultura de direitos humanos, que fosse reafirmada a educação como direito de todos e que se estabelecesse metas para a efetivação desse direito, ou seja, metas para que o sistema escolar conseguisse atender todos os grupos que até então vinham sendo excluídos desse sistema (Duck, 2005; Lourenço, 2010).

Faz-se importante também mencionar que o Conselho Federal de Psicologia instituiu, no ano 1999, o Prêmio Helena Antipoff - educação inclusiva: desafios, limites e perspectivas, publicando em 2000, no periódico Psicologia: Ciência e Profissão, os trabalhos vencedores na categoria psicólogo e na categoria estudante. Essa iniciativa do Conselho está relacionada à concentração de 5 dos artigos selecionados para esta pesquisa no ano 2000.
Essa predominância temática nos resultados deste estudo se, por um lado, indica um viés do critério adotado para a seleção de artigos - viés criado quando se decidiu pela inclusão de artigos que apresentavam no título, nas palavras-chave ou no resumo termos referentes à educação especial - por outro lado, revela que, embora o periódico tenha publicado artigos que tratam de outros aspectos da educação inclusiva, como a educação no sistema prisional, a educação em classes hospitalares, o sistema de cotas na universidade, etc., esses artigos não estão vinculados explicitamente ao tema educação inclusiva ou ao tema inclusão em seu título, em seu resumo ou nas palavras-chave, questão que merece ser melhor abordada em investigações futuras.

\section{Conclusões}

Ao encerrar a análise dos artigos selecionados, merece destaque o teor crítico dos artigos e a postura dos profissionais e pesquisadores no sentido de avaliar a atuação de psicólogos e educadores frente às demandas da educação inclusiva e de denunciar como práticas pretensamente inclusivas podem dar continuidade aos processos históricos de exclusão social.

Verificou-se que a temática das relações entre a Psicologia e a educação inclusiva se faz presente no periódico Psicologia: Ciência e Profissão, porém, aparece restrita a alguns intervalos de tempo específicos, intervalos esses que coincidem de alguma forma com momentos de mudanças nas políticas educacionais brasileiras com relação à educação inclusiva. Essa coincidência indica que a área ainda está em processo de configuração pelos psicólogos brasileiros e que essa é uma área para a qual os profissionais voltam sua atenção especialmente em momentos de mudanças no sistema educacional. 
O presente estudo não contemplou todos os artigos que tratam da educação inclusiva publicados no periódico Psicologia: Ciência e Profissão em decorrência da escolha dos critérios para a seleção de artigos. Uma nova análise do periódico certamente revelará uma ampla produção que vai além da temática da inclusão de alunos com necessidades educacionais especiais.

Embora crescente, o número de artigos que versam sobre educação inclusiva no periódico é pequeno para que sejam feitas grandes generalizações acerca da atuação do profissional na área e acerca das tendências de pesquisa sobre o tema. Pela característica da revista, de contemplar tanto relatos de pesquisa quanto relatos de experiências práticas, os artigos se dividem entre essas duas categorias. Isso, se por um lado permite ao leitor psicólogo uma visão ampla da sua profissão e da ciência que a sustenta, por outro lado, dificulta uma avaliação aprofundada tanto a respeito da produção em pesquisa quanto a respeito da atuação prática do profissional na interface com a educação inclusiva. Assim, entende-se que os resultados aqui apresentados podem ser enriquecidos com a realização de estudos similares em outros periódicos que privilegiem o registro de resultados de pesquisas e/ou com entrevistas a profissionais que estejam atuando junto a escolas e outras instituições educacionais que promovam a educação inclusiva.

\section{Érika Lourenço}

Doutorado em Educação pela Universidade Federal de Minas Gerais. Professora Adjunta II no Curso de Graduação em Psicologia e no Programa de Pós-Graduação em Psicologia da Universidade Federal de Minas Gerais - Belo Horizonte - MG - Brasil.

E-mail: erikalourenco.mail@gmail.com

\section{Ciléia Saori Hamada de Miranda}

Monitora de História da Psicologia. Graduação em andamento em Psicologia pela Universidade Federal de Minas Gerais - Belo Horizonte - MG - Brasil.

E-mail: psiufmgcileia@gmail.com

\section{Jordana Mendes Póvoa}

Graduação em andamento em Psicologia pela Universidade Federal de Minas Gerais - Belo Horizonte - MG- Brasil. E-mail: jordanapovoa@hotmail.com

Endereço para envio de correspondência:

Universidade Federal de Minas Gerais - Faculdade de Filosofia e Ciências Humanas - Departamento de Psicologia Avenida Antônio Carlos, 6627, Pampulha, Belo Horizonte - MG - Brasil. CEP: 31270-901 
Campos, R. H. F., \& Bernardes, L. H. G. (2005). A revista Psicologia: Ciência e Profissão: um registro da história recente da psicologia brasileira. Psicologia: Ciência e Profissão, 25(4), 508-525.

Costa, A. L. F., Amorim, K. M. O., \& Costa, J. P. (2010). Profissão do psicólogo no Brasil: análise da produção científica em artigos. In O. H. Yamamoto \& A. L. F. Costa (Orgs.), Escritos sobre a profissão de psicólogo no Brasil (pp. 31-58). Natal, RN: EDUFRN.

Duck, C. (2005). Educar na diversidade: material de formação docente (3a. ed.). Brasília, SP: MEC, SEESP. Recuperado em 12 setembro de 2009, do Portal MEC: http://portal.mec.gov. br/seesp/arquivos/pdf/educarnadiversidade2006.pdf

Lima, P. A. (2006). Educação inclusiva e igualdade social. São Paulo: Avercamp.

Lourenço, E. (2010). Conceitos e práticas para refletir sobre a educação inclusiva. Belo Horizonte, MG: Autêntica.

Guedes, M. C. (2010). De revista institucional a periódico científico: considerações para uma história de Psicologia:
Ciência e Profissão. Psicologia: Ciência e Profissão, 30 (Esp.), 148-155.

Gomes, W. B., \& Gauer, G. (2010). Considerações sobre a história editorial recente da revista. Psicologia: Ciência e Profissão, 30 (Esp.), 42-90.

Saldanha, A. M. (1979). Nossa revista. Psicologia: Ciência e Profissão (online). 0 . Recuperado em 17 maio 2012, de Pepsic (Periódicos Eletrônicos em Psicologia):http://pepsic. bvsalud.org/scielo.php?script =sci arttext\&pid =S1414 98931979000100001\&lng $=\mathrm{pt \& nrm}=$ iso\&tlng=pt

Souza, L. K., Gauer, G., \& Hutz, C. (2004). Publicações em psicologia do desenvolvimento em dois periódicos nacionais da década de 1990. Psico-USF, 9(1), 49-57.

Yamamoto, O. H., Souza, C., \& Yamamoto, M. E. (1999). A produção científica na psicologia: uma análise dos periódicos brasileiros no período 1990-1997. Psicologia: Reflexão e Crítica, 12(2), 549-565. 